\title{
Co-occurrence Features of Multi-scale Directional Filter Bank for Texture Charcterization
}

\author{
K. O. Cheng, N. F. Law, and W. C. Siu \\ Centre for Multimedia Signal Processing \\ Department of Electronic \& Information Engineering \\ The Hong Kong Polytechnic University \\ Hong Kong
}

\begin{abstract}
In this paper, we propose to use co-occurrence features computed from multi-scale directional filter bank (MDFB) for texture characterization. As the filter band coefficients are localized frequency components, features from co-occurrence matrices of filter bands can characterize structures of textures by describing correlation among coefficients. Our experiments show that the co-occurrence features outperform energy features considerably in texture retrieval. In particular, they significantly improve the retrieval rate for textures with weak directionality and periodicity while still maintain a high retrieval rate for regular textures as the energy features.
\end{abstract}

\section{INTRODUCTION}

Due to advanced multimedia technology, the growth of storage and distribution of images in digital form increases rapidly. To facilitate searching and access of image content from a large database such as digital libraries, content-based image retrieval (CBIR) has been introduced. Compared with text-based approach, CBIR does not require manual annotation for each image and is not limited by availability of lexicons. In CBIR, texture has widely been used as an image feature together with colour and shape, for example, homogenous texture descriptor in MPEG-7 [1]. Therefore, an effective and efficient extraction of texture features is much desired.

Many techniques of feature extraction have been developed for textures. Among them, multi-channel filtering approaches have received much attention in recent years. One of the main principles behind them is that human visual system perceives image in a scale and orientation manner [2]. Gabor filters [3] and multi-scale directional filter bank (MDFB) [4] are two of multi-channel approaches which can decompose an image into scale and directional components in a feasible way. For textures, the energy signature such as L1 norm or L2 norm of each filter band is usually computed as features. On the other hand, Wouwer et al. [5] have shown that co-occurrence features extracted in wavelet domain can improve texture characterization considerably. Using wavelets, an image is decomposed into localized frequency components. Spatial correlation of wavelet coefficients due to the structure of textures can be captured by the cooccurrence features. In this paper, we propose to use cooccurrence features calculated from MDFB for texture description. The components of MDFB are also localized but with higher angular frequency resolution than wavelet. We describe and explain the co-occurrence matrices computed from MDFB subbands for feature extraction. Experimental results in texture retrieval are provided to show the effectiveness of the proposed features for texture characterization.

This paper is organized as follows. In Section II, the MDFB adopted is introduced. Then, computation of proposed co-occurrence features from MDFB is discussed in Section III. The experimental results of the proposed features in texture retrieval and comparative studies are given in Section IV. Finally, we conclude this paper in Section V.

\section{MULTI-SCALE DIRECTIONAL FILTER BANK}

A multi-scale directional filter bank (MDFB) proposed in [4] is used to decompose a texture image into various scale and direction components. The block diagram of MDFB is illustrated in Fig. 1. First, an image is split into a lowpass and a highpass images using a lowpass filter $h_{0}[\mathbf{n}]$ with passband $[0,3 \pi / 4]$. The lowpass image is further decomposed into two bandpass images using Laplacian pyramid with the prototype filter $h_{L}[\mathbf{n}]$. The three bandpass images, which correspond to frequency bands $[\pi / 4, \pi / 2]$, $[\pi / 2,3 \pi / 4]$ and $[3 \pi / 4, \pi]$, are considered for texture characterization whereas the lowest resolution image is ignored. No feature is extracted from the lowest resolution image because characteristics of textures mainly appear in high and mid frequency ranges. For each of the three bandpass images, DFB with non-modulated structure [6] is applied to obtain directional components in the corresponding scale. In the following content, the multi-scale and multi-directional decomposition is denoted by $\left(l_{1}, l_{2}, l_{3}\right)$, where $l_{s}$ denotes the decomposition level of DFB at scale $s$. The subband at scale $s$ in direction $d$ is denoted by $x_{s, d}$. 


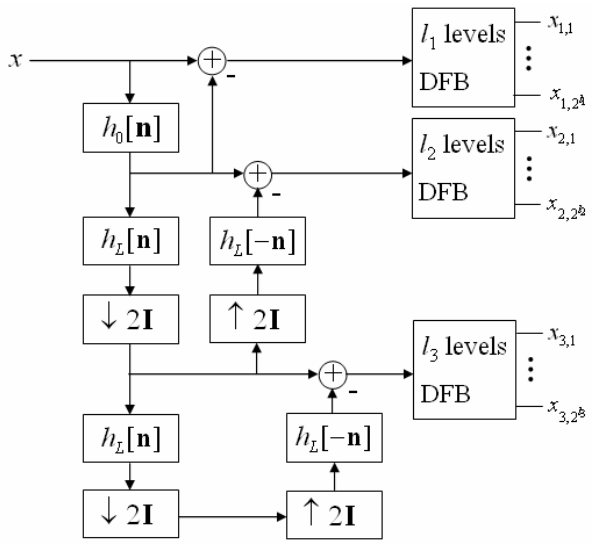

Figure 1. Block diagram of MDFB.

\section{THE PROPOSED CO-OCCURRENCE FEATURES}

Co-occurrence matrices can describe the statistics of pixel values (or coefficients) at two positions with a fixed displacement in an image (or a subband). Features computed from co-occurrence matrices can thus characterize the spatial relation between pixels (or subband coefficients) and are widely studied in texture characterization $[5,7]$. In this section, we describe how we obtain features from cooccurrence matrices in subbands of MDFB.

\section{A. Co-occurrence Matrices}

Denote the intensity function of an image and the cooccurrence matrix at distance $\delta$ in direction $\theta$ as $I(x, y)$ and $\mathrm{C}^{\delta, \theta}$ respectively. The $(i, j)$ entry of the matrix $\mathrm{C}^{\delta, \theta}$ represents the relative frequency of co-occurrence of an intensity value equal to $i$ and its neighboring intensity value at distance $\delta$ in direction $\theta$ equal to $j$. Mathematically, before normalized by total number of pairs, it is given by

$$
\begin{aligned}
& C^{\delta, \theta}(i, j)=\#\left\{\left(\left(x_{1}, y_{1}\right),\left(x_{2}, y_{2}\right)\right) \in\left(L_{x} \times L_{y}\right) \times\left(L_{x} \times L_{y}\right):\right. \\
& I\left(x_{1}, y_{1}\right)=i, I\left(x_{2}, y_{2}\right)=j, \text { where } x_{1}-x_{2}=\delta \cos \theta \text { and } \\
& y_{1}-y_{2}=\delta \sin \theta \text { or } x_{1}-x_{2}=-\delta \cos \theta \text { and } y_{1}-y_{2}= \\
& -\delta \sin \theta\}
\end{aligned}
$$

where $L_{x}$ and $L_{y}$ are horizontal and vertical domains of the image respectively. Hence, co-occurrence matrices of various distances and directions can characterize the geometric features in an image. Equation (1) can be used to compute the co-occurrence matrices in filter bands. However, filter bands usually consist of coefficients in real numbers rather than integers so quantization is required to first convert the coefficients into discrete values, i.e.,

$$
q\left(x_{s, d}\right)=\operatorname{round}\left[x_{s, d} / Q\right]
$$

where $Q$ is the quantization step size to be determined experimentally.

\section{B. Proposed Co-occurrence Features For MDFB}

In DFB, the decimations in horizontal and vertical directions are not the same. Specifically, for an $N \times N$ image with $l$ levels decomposition, the subbands either have size of $N / 2^{l-1} \times N / 2$ or $N / 2 \times N / 2^{l-1}$ after decimation. More information can be found in the direction of smaller downsampling factor, i.e. higher resolution. Therefore, cooccurrence matrix of distance one in either vertical or horizontal direction is computed for each subband in MDFB. The direction with smaller downsampling factor is selected. The co-occurrence matrix is of distance one only because significant correlation between coefficients exists only in small displacement in textures [5].

From each co-occurrence matrix, eight types of cooccurrence features proposed in [5] are calculated and analyzed for texture retrieval. Since all of them together would introduce a high dimensional feature vector, combinations of features up to two different types are investigated only. The combination which gives the highest retrieval rate in experiment should be selected as the texture features. In addition, it is possible to combine the cooccurrence features with energy signatures such as L1 norm and L2 norm given as $E k, k=1,2$ in (3) respectively for texture characterization. The retrieval performance of different combinations is studied in Section IV in detail.

$E k=\left(\frac{1}{N_{i} N_{j}} \sum_{i=1}^{N_{i}} \sum_{j=1}^{N_{j}}\left|x_{s, d}[i, j]\right|^{k}\right)^{1 / k}$

where $k=1,2$ and $N_{i} \times N_{j}$ is the size of the subband.

\section{EXPERIMENTAL RESULTS}

The retrieval performance of the proposed co-occurrence features from MDFB is studied using a texture database derived from $111512 \times 512$ texture images in Brodatz album. Each album image is split into $16128 \times 128$ non-overlapping sub-images. Sub-images from the same album image are regarded to be of the same class. This results in a database of 1776 images with 111 classes. Each image in the database is used as a query image one by one in the experiment. Before feature extraction, histogram equalization is performed to reduce the bias of intensity distribution towards sub-images from the same album image. The similarity between two texture images, image 1 and image 2, is defined as weighted Euclidean distance between their feature vectors [3], i.e.,

$D\left(\mathbf{f}^{(1)}, \mathbf{f}^{(2)}\right)=\left(\sum_{i=1}^{N}\left(f_{i}^{(1)}-f_{i}^{(2)}\right)^{2} / \sigma_{i}^{2}\right)^{1 / 2}$

where $\mathbf{f}^{(k)}$ is the feature vector of texture image $k, f_{i}^{(k)}$ is the $i$-th element of $\mathbf{f}^{(k)}$ and $\sigma_{i}$ is the standard deviation of the $i$-th element of the feature vectors in the entire database. 
MDFB of decomposition $(3,4,3)$ is used. The filters in DFB are implemented using the ladder structure of finite impulse response [8]. The half-band lowpass filter used in Laplacian pyramid is a binomial filter of length 15 . It has been shown that this specification of MDFB gives a relatively high retrieval rate compared with others for energy signatures [4]. In order to optimize the quantization step size $Q$ for subband coefficients mentioned in Section III.A, the experiment is performed several times with different values of $Q$. It is found that the retrieval rate converges to a maximum around $Q=4$ for all the co-occurrence features. Hence, we set $Q=4$ throughout the experiment.

\section{A. Comparison with energy signatures}

The overall average retrieval rate of combined features up to two different types, which include co-occurrence features as well as energy signatures, is summarized in Table I. The average retrieval rates using energy signatures E1 and E2 are $67.2 \%$ and $67.4 \%$ over the entire database respectively. However, with the use of co-occurrence features $\mathrm{C} 3$ and $\mathrm{C} 8$, the average retrieval rate increases to $72.3 \%$, which is the highest among all the combinations. Also, combined with $\mathrm{C} 8$, the average retrieval rates of $\mathrm{E} 1$ and $\mathrm{E} 2$ are increased by $4.5 \%$ and $4.4 \%$ respectively.

The use of co-occurrence features actually improves retrieval performance by increasing the complexity in feature extraction. As the computation of co-occurrence matrices can be realized by bit-shifting (for quantization), additions and indexing, we can assume that the computational complexity of co-occurrence matrices is similar to that of energy features. The extra computations are hence due to features calculation from co-occurrence matrices. However, feature extraction for database images is usually performed offline so the retrieval time would only be increased in a very little amount.

\section{B. Comparison with co-occurrence features from DWT}

Comparative study has been performed with the cooccurrence features extracted from 4 levels D-spline discrete wavelet transform (DWT) of order 2. In the DWT algorithm, greedy algorithm has been applied to select the cooccurrence feature types for retrieval. It was found that five different feature types gave the best performance. This resulted in 60 features for each texture image. The results are summarized in Table II. It can be seen that the best result for co-occurrence features from MDFB as shown in Table I is $5.4 \%$ higher than that from the DWT while the number of features required is kept about the same. The improvement is due to the higher angular resolution provided by DFB.

\section{Further analysis}

In order to further study the co-occurrence features, the average retrieval rate for each texture class, which is provided in Table III, is examined. It is found that the retrieval rate is improved for most of the texture classes. The improvement is significant for texture classes D002, D005, D067 and D074. As can be seen in Fig.2, all of them lack of directionality and periodicity. This implies that cooccurrence features of MDFB can characterize regular textures at various scales and orientations whereas still account for the randomness of irregular textures. This is due to the fact that the co-occurrence features provide a statistical texture description, which complement the spectral texture description in the MDFB.

\section{CONCLUSION}

Co-occurrence features for multi-scale directional filter bank have been proposed for texture characterization. The co-occurrence features can describe the spatial correlation of subband coefficients. Thus, the geometrical arrangement of texture features in various scale and direction can be characterized. Experiments show that the use of cooccurrence features results in higher retrieval performance than the energy features especially for the irregular textures. Furthermore, the MDFB is superior than DWT for cooccurrence features extraction. As a result, the proposed features provide both a statistical and a spectral texture description which effectively describe the structural features for both regular and irregular textures.

TABLE I. RETRIEVAL RATE (\%) FOR DIFFERENT COMBINATIONS UP TO TWO TYPES OF CO-OCCURRENCE FEATURES AND/OR ENERGY FEATURES. A,B

\begin{tabular}{|c|c|c|c|c|c|c|c|c|c|c|c|}
\hline \multirow{2}{*}{\multicolumn{2}{|c|}{ Feature }} & \multicolumn{8}{|c|}{ Co-occurrence features ${ }^{\mathrm{c}}$} & \multicolumn{2}{|c|}{ Energy signature } \\
\hline & & $\mathrm{C} 1$ & $\mathrm{C} 2$ & C3 & $\mathrm{C} 4$ & $\mathrm{C} 5$ & C6 & $\mathrm{C} 7$ & $\mathrm{C} 8$ & E1 & E2 \\
\hline \multirow{4}{*}{$\begin{array}{l}\text { Co- } \\
\text { occurrence } \\
\text { feature }\end{array}$} & $\mathrm{C} 2$ & - & 59.2 & 66.6 & 64.9 & 58.7 & 46.4 & 59.6 & 69.3 & 67.1 & 67.7 \\
\hline & $\mathrm{C} 4$ & - & - & - & 65.2 & 64.5 & 58.1 & 64.5 & 71.6 & 67.5 & 68.4 \\
\hline & $\mathrm{C} 5$ & - & - & - & - & 57.9 & 49.1 & 60.7 & 69.8 & 67.0 & 67.9 \\
\hline & C6 & - & - & - & - & - & 30.5 & 47.3 & 60.2 & 61.1 & 61.1 \\
\hline \multirow{2}{*}{$\begin{array}{l}\text { Energy } \\
\text { signature }\end{array}$} & E1 & - & - & - & - & - & - & - & - & 67.2 & 67.6 \\
\hline & E2 & - & - & - & - & - & - & - & - & - & 67.4 \\
\hline
\end{tabular}

a. Due to symmetry of the table, only half of retrieval rate is shown.

b. The use of combination of features of the same type is equivalent to the use of features of that type without combination. c. Co-occurrence features C1-C8 refer to inertia, total energy, entropy, local homogeneity, max. probability, cluster shade, cluster prominence and information measure of correlation respectively. 
TABLE II. COMPARISON OF RETRIEVAL RESULTS

\begin{tabular}{|l|l|l|}
\hline & Best proposed features & $\begin{array}{l}\text { Co-occurrence features } \\
\text { from DWT }\end{array}$ \\
\hline Retrieval rates (\%) & 72.3 & 66.9 \\
\hline No. of features & 64 & 60 \\
\hline
\end{tabular}

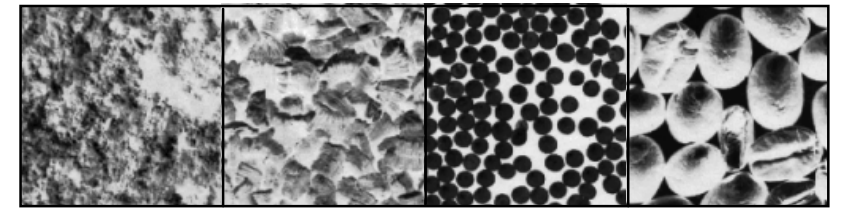

Figure 2. Samples for classes D002, D005, D067 and D074 (left to right).

TABLE III. AVERAGE RETRIEVAL RATE (\%) FOR EACH TEXTURE CLASSES WITH DIFFERENT FEATURES.

\begin{tabular}{|c|c|c|c|c|c|c|c|c|c|c|c|c|c|c|}
\hline \multirow[t]{2}{*}{ Class } & \multicolumn{4}{|c|}{ Features } & \multirow[t]{2}{*}{ Class } & \multicolumn{4}{|c|}{ Features } & \multirow[t]{2}{*}{ Class } & \multicolumn{4}{|c|}{ Features } \\
\hline & E1 & E2 & E2,C8 & C3,C8 & & E1 & E2 & E2,C8 & C3,C8 & & E1 & E2 & E2,C8 & C3,C8 \\
\hline D001 & 92 & 93 & 95 & 95 & D002 & 59 & 60 & 66 & 95 & D003 & 46 & 48 & 58 & 64 \\
\hline D004 & 100 & 100 & 100 & 99 & D005 & 79 & 76 & 77 & 99 & D006 & 100 & 100 & 100 & 100 \\
\hline D007 & 34 & 35 & 45 & 45 & D008 & 91 & 90 & 99 & 45 & D009 & 56 & 54 & 60 & 61 \\
\hline D010 & 73 & 73 & 77 & 76 & D011 & 100 & 100 & 100 & 76 & D012 & 82 & 83 & 80 & 82 \\
\hline D013 & 40 & 39 & 40 & 42 & D015 & 48 & 47 & 46 & 42 & D016 & 100 & 100 & 100 & 100 \\
\hline D017 & 100 & 100 & 100 & 100 & D018 & 96 & 97 & 96 & 100 & D019 & 84 & 84 & 94 & 97 \\
\hline D020 & 100 & 100 & 100 & 100 & D021 & 100 & 100 & 100 & 100 & D022 & 48 & 49 & 52 & 66 \\
\hline D023 & 43 & 45 & 60 & 59 & D024 & 89 & 88 & 89 & 59 & D025 & 48 & 54 & 56 & 55 \\
\hline D026 & 84 & 88 & 93 & 91 & D027 & 41 & 41 & 54 & 91 & D028 & 64 & 64 & 68 & 71 \\
\hline D029 & 100 & 100 & 100 & 98 & D030 & 46 & 41 & 44 & 98 & D031 & 40 & 37 & 52 & 56 \\
\hline D032 & 63 & 64 & 74 & 79 & D033 & 75 & 82 & 88 & 79 & D034 & 99 & 100 & 100 & 99 \\
\hline D035 & 57 & 58 & 61 & 64 & D036 & 41 & 42 & 46 & 64 & D037 & 93 & 97 & 99 & 98 \\
\hline D038 & 64 & 66 & 71 & 73 & D039 & 42 & 42 & 54 & 73 & D040 & 48 & 43 & 56 & 61 \\
\hline D041 & 63 & 63 & 83 & 89 & D042 & 34 & 35 & 41 & 89 & D043 & 25 & 23 & 25 & 26 \\
\hline D044 & 23 & 22 & 25 & 29 & D045 & 21 & 18 & 23 & 29 & D046 & 97 & 97 & 88 & 88 \\
\hline D047 & 95 & 99 & 98 & 96 & D048 & 95 & 93 & 90 & 96 & D049 & 100 & 100 & 100 & 100 \\
\hline D050 & 65 & 59 & 70 & 65 & D051 & 79 & 73 & 73 & 65 & D052 & 100 & 100 & 100 & 100 \\
\hline D053 & 100 & 100 & 100 & 100 & D054 & 49 & 54 & 59 & 100 & D055 & 100 & 100 & 100 & 100 \\
\hline D056 & 100 & 100 & 99 & 99 & D057 & 100 & 100 & 100 & 99 & D058 & 17 & 18 & 22 & 22 \\
\hline D059 & 21 & 18 & 32 & 30 & D060 & 39 & 38 & 43 & 30 & D061 & 38 & 33 & 40 & 39 \\
\hline D062 & 38 & 39 & 40 & 40 & D063 & 21 & 24 & 33 & 40 & D064 & 98 & 98 & 100 & 100 \\
\hline D065 & 100 & 100 & 100 & 100 & D066 & 77 & 74 & 91 & 100 & D067 & 39 & 42 & 61 & 60 \\
\hline D068 & 98 & 100 & 100 & 99 & D069 & 24 & 25 & 30 & 99 & D070 & 75 & 76 & 81 & 78 \\
\hline D071 & 25 & 25 & 29 & 32 & D072 & 38 & 38 & 38 & 32 & D073 & 46 & 50 & 60 & 59 \\
\hline D074 & 63 & 58 & 90 & 92 & D075 & 80 & 84 & 93 & 92 & D076 & 99 & 97 & 100 & 100 \\
\hline D077 & 100 & 100 & 100 & 100 & D078 & 100 & 100 & 100 & 100 & D079 & 98 & 98 & 100 & 100 \\
\hline D080 & 100 & 100 & 100 & 100 & D081 & 100 & 100 & 100 & 100 & D082 & 100 & 100 & 100 & 100 \\
\hline D083 & 100 & 100 & 100 & 100 & D084 & 100 & 100 & 100 & 100 & D085 & 98 & 99 & 100 & 100 \\
\hline D086 & 81 & 81 & 85 & 85 & D087 & 86 & 87 & 91 & 85 & D088 & 50 & 50 & 65 & 66 \\
\hline D089 & 39 & 39 & 52 & 53 & D090 & 29 & 26 & 31 & 53 & D091 & 30 & 29 & 29 & 30 \\
\hline D092 & 80 & 79 & 79 & 79 & D093 & 85 & 88 & 88 & 79 & D094 & 70 & 79 & 91 & 89 \\
\hline D095 & 100 & 100 & 100 & 100 & D096 & 69 & 76 & 85 & 100 & D097 & 23 & 22 & 21 & 23 \\
\hline D098 & 51 & 52 & 66 & 70 & D099 & 27 & 23 & 30 & 70 & D100 & 44 & 46 & 62 & 54 \\
\hline D101 & 89 & 88 & 79 & 73 & D102 & 93 & 93 & 87 & 73 & D103 & 56 & 56 & 52 & 53 \\
\hline D104 & 48 & 48 & 54 & 55 & D105 & 65 & 65 & 71 & 55 & D106 & 55 & 54 & 50 & 49 \\
\hline D107 & 25 & 25 & 30 & 31 & D108 & 37 & 36 & 46 & 31 & D109 & 40 & 41 & 44 & 43 \\
\hline D110 & 50 & 50 & 59 & 64 & D111 & 41 & 43 & 58 & 64 & D112 & 54 & 51 & 56 & 57 \\
\hline
\end{tabular}

\section{ACKNOWLEDGMENT}

This work is supported by RGC Grant PolyU 5222/03E and the Centre for Multimedia Signal Processing (A452), the Hong Kong Polytechnic University.

\section{REFERENCES}

[1] T. Sikora, "The MPEG-7 visual standard for content description - an overview," IEEE Trans. Circuits Syst. Video Technol., vol. 11, pp. 696702, June 2001.

[2] J. G. Daugman, "Uncertainty relation for resolution in space, spatial frequency, and orientation optimized by two-dimensional visual cortical filters," J. Opt. Soc. Am. A, vol. 2, pp. 1160-1169, July 1985.
[3] B. S. Manjunath and W. Y. Ma, "Texture features for browsing and retrieval of image data," IEEE Trans. Pattern Anal. Machine Intell., vol. 18, pp. 837-842, August 1996.

[4] W. Y. Chan,N. F. Law, and W. C. Siu, "Multiscale feature analysis using directional filter bank,” ICICS-PCM'03, vol.2, pp.822-826, Dec 2003.

[5] G. V. D. Wouwer, P. Scheunders, and D. V. Dyck, "Statistical texture characterization from discrete wavelet representations," IEEE Trans. Image Processing, vol. 8, pp. 592-598, April 1999.

[6] S.-I. Park, M. J. T. Smith, and R. M. Mersereau, "A new directional filter bank for image analysis and classification," ICASSP'99, vol. 3, pp. 1417-1420, March 1999.

[7] A. Busch, W. W. Boles and S. Sridharan, "Logarithmic quantisation of wavelet coefficients for improved texture classification performance," ICASSP'04, vol. 3, pp. III-569-572, May 2004.

[8] S.-M. Phoong, C. W. Kim, P. P. Vaidyanathan, and R. Ansari, "A new class of two-channel biorthogonal filter banks and wavelet bases," IEEE Trans. Signal Processing, vol. 43, no. 3, pp. 649-665, March 1995. 